\title{
Performance Analysis of a Cellular Networks Using Power Control Based Frequency Reuse Partitioning
}

\author{
Mustafa Habibu Mohsini`, Seung-Yeon Kim, Choong-Ho Cho
}

\begin{abstract}
This paper focuses on evaluating the performance of a cellular network using power control based frequency reuse partitioning (FRP) in downlink (DL). In our work, in order to have the realistic environment, the spectral efficiency of the system is evaluated through traffic analysis, which most of the previous works did not consider. To further decrease the cell edge user's outage, the concept of power ratio is introduced and applied to the DL FRP based cellular network. In considering network topology, we first divide the cell coverage area into two regions, the inner and outer regions. We then allocate different sub-bands in the inner and outer regions of each cell. In the analysis, for each zone ratio, the performance of FRP system is evaluated for the given number of power ratios. We consider performance metrics such as call blocking probability, channel utilization, outage probability and effective throughput. The simulation results show that there is a significant improvement in the outage experienced by outer UEs with power control scheme compared to that with no power control scheme and an increase in overall system throughput.
\end{abstract}

Key Words : Inter-Cell Interference (ICI), Interference Management, Frequency Reuse Partitioning (FRP).

\section{I . Introduction}

\subsection{Motivation and Related Work}

In order to achieve high system capacity and simplify the radio network planning, most OFDMA based fourth-generation (4G) systems (such as WiMAX) IEEE $802.16^{[1]}$ and 3GPP-LTE ${ }^{[2]}$ are targeting the frequency reuse factor of $1(\mathrm{FRF}=1)$. FRF 1 implies that the base stations in cells share all feasible time-frequency resource blocks (RBs) simultaneously. However, in OFDMA multi-cell systems deployed with frequency reuse 1, co-channel interference (CCI) occurs due to the simultaneous use of the same channel or subcarrier by different users in the neighboring cells. There is a severe increase in the outage experienced by the users especially cell edge users who suffer severe performance degradation due to the very strong CCI from neighboring cells and the out-of-cell transmissions which leads to poor cell coverage. Thus to mitigate CCI, FRF of three or more has generally been employed.

Interference mitigation technique by controlling the degree of frequency reuse is one of the most important techniques for interference mitigation ${ }^{[3]}$. In [4], several inter-cell interference coordination (ICIC) schemes have been proposed, one among them being Frequency Reuse Partitioning (FRP). In [5], FRP has been proposed for the OFDMA based IEEE $802.16 \mathrm{~m}$ and 3GPP-LTE systems as ICIC technique. In addition, through using different cluster sizes, FRP has been suggested in [6] to be

※ This research was supported by Basic Science Research Program through the National Research Foundation of Korea(NRF) funded by the Ministry of Education, Science and Technology(2010-0025125)

- First Author : Korea University, Department of Computer and Information Science, mustafa30@korea.ac.kr, 학생회원

- Corresponding Author : Korea University, Department of Computer and Information Science, chcho@korea.ac.kr, 종신회원

* Korea University, Department ofComputer and Information Science, kimsy8011@ korea.ac.kr 논문번호 : KICS2015-01-004, Received January 08, 2015; Revised February 24, 2015; Accepted March 17, 2015 
a very useful technique to achieve high spectrum efficiency in cellular systems.

The main idea of FRP is to divide the cell into concentric regions and to further reduce ICI; each region is allocated different sub-bands. The same set of sub-bands is not used in the neighboring cell edges.

The performance of the FRP approach has been reported in [7], [8] and [9]. In [7], the performance of the reuse partitioning was evaluated and analytical model for the FRP based system was proposed. In [8], the power division reuse partitioning scheme and successive interference cancellation was proposed. The performance of power control to an improved fractional frequency reuse (IFFR) scheme was investigated in [9]. However, [8] did not consider the effect of power control. In addition, in [7] and [8], the performance was evaluated in the uplink (UL) transmission where user equipments (UEs) transmit with the same average power and hence the effect relies only on the path loss and shadowing effect. On the other hand, [8] and [9] did not take into account the traffic analysis which is necessary part for the evaluation of the spectral efficiency of the system.

\subsection{Contribution and Organization}

To the best of our knowledge, we have seen that there is no work that discussed the performance of FRP taking into consideration the analysis of the traffic in the network for downlink (DL) transmission and power control. Traffic analysis provides realistic environment as it provides the details of the channels used and the accepted calls. In contrast with that of [7], the performance of our system not only relies on the path loss and shadowing effect but also the effect of the transmission power of the BS which takes different values for inner and outer regions. In this paper, we therefore added the traffic analysis part in the power control based two-tier FRP system. In addition, for each zone ratio, different power ratios are simulated and the system performance is evaluated. The results of the channel utilizations and call blocking probabilities for the inner UEs, outer UEs and overall UEs are presented. Furthermore, outer UEs outage, inner UEs outage in terms of offered load and effective throughput in terms of the outage probability are also presented.

The remainder of this paper is organized as follows: Section II outlines the details of system model. In Section III, the proposed algorithm is described in detail, and the performance metrics are discussed in Section IV. Simulation results and analysis are discussed in Section V. Finally, Section $\mathrm{V}$ concludes the paper.

\section{System Model}

\subsection{Model Descriptions}

As shown in Figure 1, we consider a system topology of a two-tier network with 19 hexagonal macrocells of equal sizes. It is assumed that the BS of each cell is positioned at the center of the cell and it is represented by small dots in each cell. In addition, each cell is further partitioned into two regions: the inner region and the outer region of concentric radii $R$ and $r$ respectively. Through Received Signal Strength (RSS) which is measured and reported by UE, the distance between the BS and UE is estimated. Accordingly, the zone ratio, $\varepsilon$ is defined as the ratio of the inner zone area to the overall cell area or simply the fraction area of the inner zone as shown in (1)

$$
\varepsilon=\frac{\pi^{*} r^{2}}{\pi^{*} R^{2}}=\left(\frac{r}{R}\right)^{2} .
$$

The total spectrum is divided into three sub-bands; $F_{0}, F_{1}$ and $F_{2}$. To further enhance the system capacity through interference mitigation, different sub-bands are allocated for the outer regions while the remaining sub-bands are reused in the inner regions of each cell avoiding the sub-band located in their respective outer regions. In other words, users in the outer region have exclusive use of the outer channels and users in the inner region use the remaining channels not used by the outer users. 


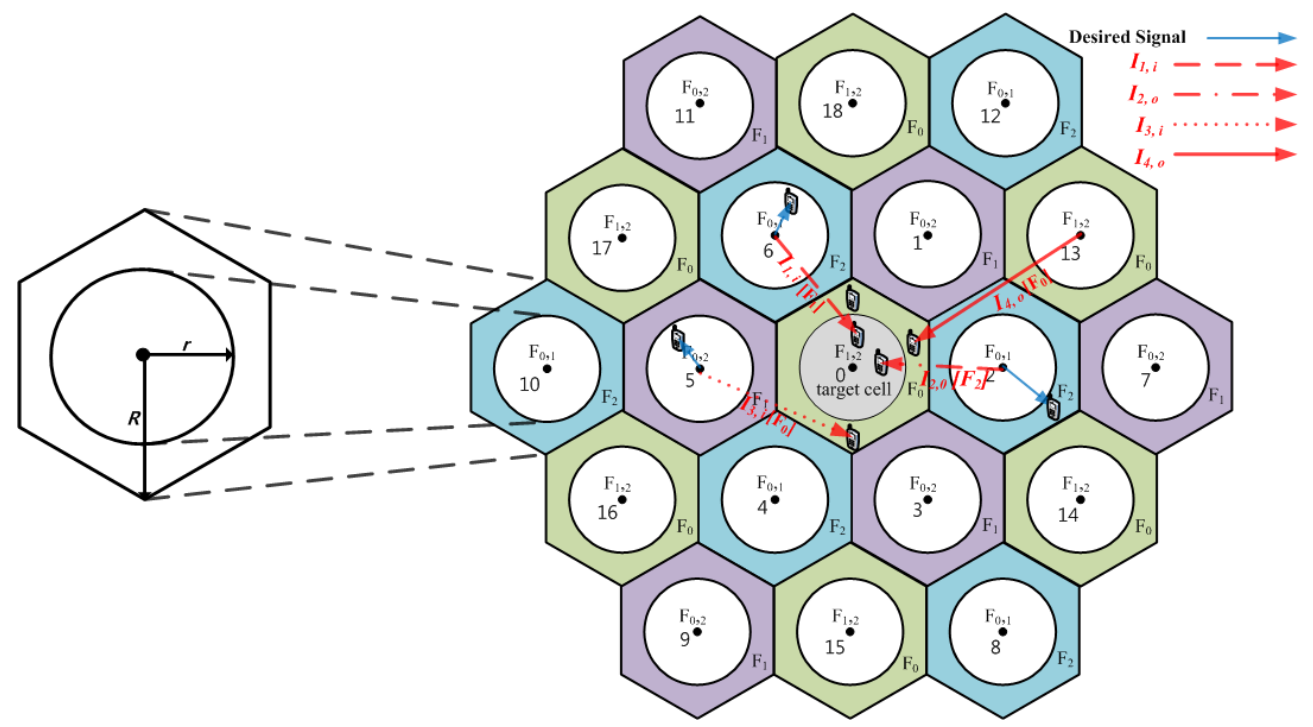

Fig. 1. The system topology and interference effect in the FRP system.

\subsection{Recieved Signal Power Model}

We assume that the received signal power, $P_{k, z}$ at user is

$$
P_{k, z}=P_{t}^{k} r_{z}^{-\alpha} L_{z}
$$

where $P_{t}^{k}$ refers to transmit power of the target cell, $B S_{0}$ i.e. cell 0 in region $k$. Throughout our paper, we refer $k=(i, o)$ as the region of transmission in the cell where $\mathrm{i}$ for inner region and o for outer region, $r_{z}$ is the distance between the $B S, z$ and user, $\alpha$ is path loss exponent, and $L_{z}$ being the shadowing effect with log-normal distribution with zero-mean and a standard deviation of $\sigma$.

\subsection{Traffic Model}

In this section, we describe the system traffic model. For the DL transmission, we assume that only one channel which is channelized from resource elements or sub-carriers can be assigned to $\mathrm{UE}$ from $\mathrm{BS}^{[11]}$. We also assume that there are a total of $C$ channels in each cell and that each of the sub-bands $F_{0}, F_{1}$ and $F_{2}$ has total outer channels, $C_{o}=C / 3$ and total inner channels, $C_{i}=C-C_{o}$. The new call is scheduled to get serving on one channel only whenever the channel is available. If the channel is not available, the call is blocked.

In addition, the traffic model has the following assumptions:

The call inter arrival time is exponentially distributed with mean $1 / \lambda$ seconds per cell

For the inner region, the call inter arrival rate is $\lambda_{i}$ and that of the outer region is $\lambda_{o}$ and they are related according to equations (3) and (4) below

$$
\begin{gathered}
\lambda_{i}=\varepsilon \lambda \\
\lambda_{o}=(1-\varepsilon) \lambda,
\end{gathered}
$$

The call serving/holding time is exponentially distributed with mean $1 / \mu$ seconds $^{[10]}$.

\section{Proposed Algorithm for Frequency Reuse Partitioning (FRP)}

In this section, we discuss the channel allocation procedure, power control, interference types and the signal to interference ratio (SIR). The proposed power control based FRP system is explained.

\subsection{Channel Allocation Procedure}

In this section, we introduce the channel 
allocation procedure for the FRP. When a new call first arrives, it requests a channel. Note that the request of the channel depends on the region upon which the call arrives i.e. in the inner or outer region. If the call arrives in the inner region of the cell, it requests a channel from the inner region and if the call arrives in the outer region of the cell, it requests a channel from the outer region. If the channel is available, then this channel is assigned to the UE while other UEs in the same cell cannot use the same channel. Upon completion of its transmission, the assigned UE releases the channel so that it becomes available to other UEs. On the other hand, when all the channels are fully utilized, the newly arriving call is rejected and we call this as initial call block. The channel selection procedure is shown in Table 1.

\section{Table 1. Channel Allocation}

\begin{tabular}{|l|}
\hline \multicolumn{1}{|c|}{ Channel Selection Procedure Algorithm } \\
\hline Call Arrival \\
Case 1:A UE arrives in the inner region \\
UE requests a channel \\
If a channel is available \\
Accept the call and assign inner channel \\
Else \\
$\quad$ Reject the call \\
Case 2:A UE arrives in the outer region \\
UE requests a channel \\
If a channel is available \\
Accept the call and assign outer channel \\
Else \\
$\quad$ Reject the call
\end{tabular}

\subsection{Power Control}

In this section, we propose the power control scheme to improve the system throughput of FRP system. To do this, we first define the power ratio. In power ratio, the BS transmit power is controlled according to the user location.

Let $P_{t}^{i}$ and $P_{t}^{o}$ be the $B S$ transmit powers in the inner and outer regions of the cell, respectively, we define power ratio, $\beta$ as in (5)

$$
\beta=\frac{P_{t}^{i}}{P_{t}^{o}} .
$$

When the transmission power level is the same in the inner and outer regions (i.e. $\beta=1$ ), we refer this case as no power control (no PC) scheme. When the inner and outer regions transmit with different power levels (i.e. $\beta \neq 1$ ), we refer this case as power control (PC) scheme.

\subsection{Co-channel Interference (CCI) Types}

Depending on whether the interference originated from transmission to inner user or outer user owing to the neighboring cells, the interference may be one of the four types.

Suppose that $I_{l, k}$ is the interference with $l=1,2,3,4$ being interference types and $k$ denote whether the interference originated from inner or outer regions of the neighboring cells to the target cell, the four types of interference due to $\mathrm{CCI}$ are described below:-

- $I_{1, i}$ : Interference originating from inner region of the neighboring cell to the inner region of the $B S_{0}$ for users using the same channel in these cells

- $I_{2, o}$ : Interference originating from outer region of the neighboring cell to the inner region of the $B S_{0}$ for users using the same channel in these cells

- $I_{3, i}$ : Interference originating from inner region of the neighboring cell to the outer region of the $B S_{0}$ for users using the same channel in these cells.

- $I_{4, o}$ : Interference originating from outer region of the neighboring cell to the outer region of the $B S_{0}$ for users using the same channel in these cells.

Though there are many co-channel interfering signals from different neighboring cells, to provide a clear understanding of these interference configurations, only four cells which show the four types of interference are presented in Figure 1.

In addition, depending on weather the UE in $B S_{0}$ is using an inner channel or an outer channel, there are two cases of interference configurations.

- Case 1: UE uses an inner channel. In this case, 
assume that the UE arrived in the inner zone of the $B S_{0}$ and use a channel in $F_{1}$. There will be interferences received from neighboring cells 2,4 , $6,8,10,12,13,14,15,16,17$ and 18 from the inner channels of these cells. There will be other interferences from neighboring cells 1, 3, 5, 7, 9 and 11 from the outer channels of these cells.

- Case 2: UE uses an outer channel. In this case, assume that the UE arrived in the outer zone of the $B S_{0}$ and use a channel in $F_{0}$, there will be interferences received from neighboring cells 1,2 , $3,4,5,6,7,8,9,10,11$, and 12 from the inner channels of these cells and other interferences from neighboring cells $13,14,15,16,17$ and 18 from the outer channels of these cells.

Summary of four types of interference from any user served by any of the neighboring cells to the user served by the target cell are presented in Table II, in which the notations used are the same as the one presented in Figure 1.

Table 2. Interference types from neighboring cells

\begin{tabular}{|c|c|c|c|}
\hline \multirow{2}{*}{$\begin{array}{c}\text { Interference } \\
\text { Type }\end{array}$} & $\begin{array}{c}\text { Channel } \\
\text { used by } \\
\text { UE in the } \\
\text { target cell }\end{array}$ & $\begin{array}{c}|c| \\
\text { Orst-tier } \\
\text { cells }\end{array}$ & Second-tier cells \\
\hline \multirow{2}{*}{$I_{1, i}$} & $F_{1}$ & $2,4,6$ & $8,10,12,13, \ldots, 18$ \\
\cline { 3 - 4 } & $F_{2}$ & 1.3 .5 & $7,9,11,13, \ldots, 18$ \\
\hline \multirow{2}{*}{$I_{2, o}$} & $F_{1}$ & $1,3,5$ & $7,9,11$ \\
\cline { 3 - 4 } & $F_{2}$ & $2,4,6$ & $8,10,12$ \\
\hline$I_{3, i}$ & $F_{0}$ & All & $7, \ldots, 12$ \\
\cline { 3 - 4 } & & None & $13, \ldots, 18$ \\
\hline$I_{4, o}$ & & & \\
\hline
\end{tabular}

\subsection{SIR Formulation}

We assume that the system interference is only limited to CCI, so noise and other kinds of interferences are negligible. The signal to interference ratio (SIR), $\gamma_{k}$ for the DL transmission to user from $B S_{0}$ in region $k$ of the cell is given by

$$
\gamma_{k}=\frac{P_{k, 0}}{P_{t}^{i} \sum_{z \in Z_{i}} r_{z}^{-\alpha} L_{z} \chi_{z}+P_{t}^{o} \sum_{z \in Z_{o}} r_{z}^{-\alpha} L_{z} \chi_{z}},
$$

where $\chi$ is the interference index taking value of 1 when the interference occurs or 0 when the interference does not occur, and $Z_{*}(*=i, o)$ is the set of co-channel cells.

It is important to note that a transmission in a cell inner region or cell outer region that is assigned a specific channel may cause interference to both inner users and outer users of the other cells that are assigned the same channel.

\section{System Performance Measurement}

\subsection{Call Blocking Probability}

Let $N_{f, k}$ and $N_{t, k}$ be the number of failed calls and total number of call trials in region $k$ of the cell respectively; the inner, outer and total call blocking probabilities; $P_{b, i}, P_{b, o}$ and $P_{b}$ are respectively given by equations (7), (8) and (9)

$$
P_{b, i}=\frac{N_{f, i}}{N_{t, i}}
$$

$$
P_{b, o}=\frac{N_{f, o}}{N_{t, o}}
$$

and

$$
P_{b}=P_{b, i}+P_{b, o}
$$

\subsection{Channel Utilization}

The channel utilization defines not only the channel occupancy ratio but also the probability of interference occurrence that the neighboring cells will suffer from the target/serving cell.

Let $S$ be simulation time and the total served time of succeeded calls in region $k$ be $T_{k}$, then the inner channel utilization, $\rho_{i}$ and the outer channel utilization, $\rho_{o}$ and the overall channel utilization, $\rho$ are respectively given as

$$
\begin{aligned}
& \rho_{i}=\frac{T_{i}}{S^{*} C_{i}}, \\
& \rho_{o}=\frac{T_{o}}{S^{*} C_{o}},
\end{aligned}
$$


and

$$
\rho=\frac{T_{i}+T_{o}}{S^{*} C}
$$

\subsection{Outage Probability}

We define the outage probability, $\eta_{k}$ in either of the region of the cell as the probability that the user's SIR in region $k, \gamma_{k}$ falls below the SIR threshold, $\gamma_{\text {threshold }}$ and is given as

$$
\eta_{k}=P\left(\gamma_{k}<\gamma_{\text {threshold }}, k=i, o\right)
$$

The total outage probability, $\eta$ is calculated by considering the outage of accepted calls in each region of the cell and is given as

$$
\eta=\frac{\lambda_{i}\left(1-P_{b, i}\right) \eta_{i}+\lambda_{o}\left(1-P_{b, o}\right) \eta_{o}}{\lambda_{i}\left(1-P_{b, i}\right)+\lambda_{o}\left(1-P_{b, o}\right)},
$$

where $\lambda_{i}$ and $\lambda_{o}$ are the same as in (3) and (4) respectively, $\left(1-P_{b, i}\right)$ and $\left(1-P_{b, o}\right)$ give the number of accepted inner calls and that of outer calls respectively.

\subsection{Effective Throughput}

The throughput of the FRP based cellular system is measured by the effective throughput and is obtained from the effective carried load per cell. From the obtained call blocking probability and the outage probability, we calculate the effective throughput, $T$ for a given offered load, $\lambda / \mu$ as

$$
T=\left[\lambda_{i}\left(1-P_{b, i}\right)\left(1-\eta_{i}\right)+\lambda_{o}\left(1-P_{b, o}\right)\left(1-\eta_{o}\right)\right] / \mu,
$$

where $\left(1-\eta_{i}\right)$ and $\left(1-\eta_{o}\right)$ define the probability of no outage in the inner and outer regions respectively.

\section{Simulation Results and Analysis}

The cellular system being simulated is composed of 19 two-tier hexagonal cells in which UEs are assumed to be uniformly distributed within a cell's coverage area. The
UEs access the channels randomly and the assignment of the channel to the UE follows the channel allocation procedure described in section III. For all of the simulation results, it is assumed that $C=51, C_{o}=17$ and $C_{i}=34$. The radius of the cell is normalized and assumed to be one. In addition, the normalized inner zone radius, $r$ is assumed to take values of $0.6,0.7$ and 0.8 . For $r=0.6,0.7$ and 0.8 , the inner regions occupies $36 \%$, $49 \%$ and $64 \%$ of the total region of the cell respectively. Other additional simulation parameters are presented and described in Table III.

We evaluate and analyze the simulation results of call blocking probability, the channel utilization, outage probability and the effective throughput versus normalized offered load when $r=0.6,0.7$ and 0.8 . The performance analysis of power control based FRP is done by examining the

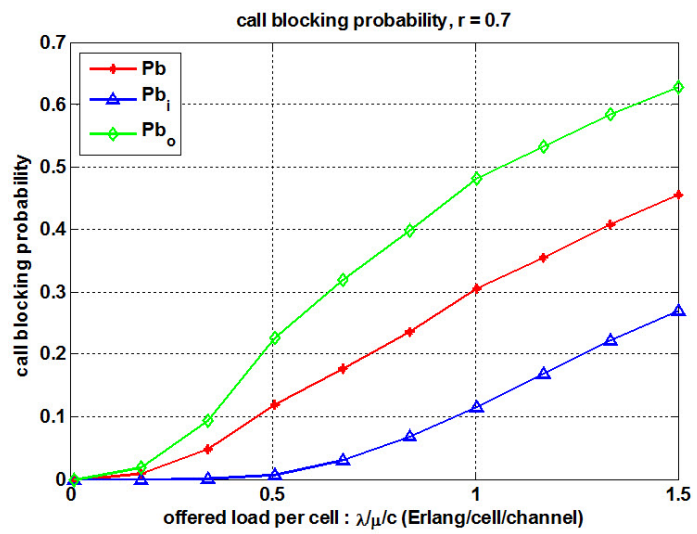

Fig. 2. Channel Utilization versus offered load with $\mathrm{r}=0.7$.

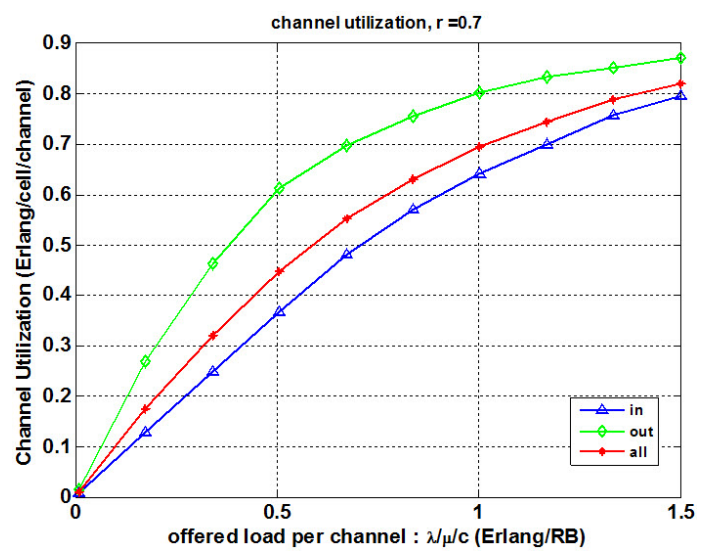

Fig. 3. Call blocking probability versus offered load with $\mathrm{r}=0.7$. 
Table 3. Simulation Parameters

\begin{tabular}{|l|l|}
\hline \multicolumn{1}{|c|}{ Parameters } & \multicolumn{1}{c|}{ Values } \\
\hline BS transmit Power & $15 \mathrm{~W}, 25 \mathrm{~W}, 40 \mathrm{~W}$ \\
\hline Number of cells & Two-tier 19 cells \\
\hline Cell radius & $1000 \mathrm{~m}$ \\
\hline Call inter arrival time & Exponential distribution \\
\hline Call serving time & Exponential distribution \\
\hline User distribution & Uniform distribution \\
\hline Path loss exponent, $\alpha$ & 4 \\
\hline Total Channel, $C$ & 51 \\
\hline Normalized $r$ & $0.6,0.7,0.8$ \\
\hline$\gamma_{\text {threshold }}$ & $-6 \mathrm{~dB}$ \\
\hline Standard deviation, $\sigma$ & $8 \mathrm{~dB}$ \\
\hline
\end{tabular}

system performance change in each of the zone ratio for the range of the given power ratio, $\varepsilon$. In our work, we compare the performance of proposed PC scheme to the one with no PC scheme. Figure 2 and Figure 3 plot the results of channel utilization and call blocking probability respectively with respect to the offered load, when $r=0.7$.

The call blocking probabilities of the inner UEs, the outer UEs and the overall UEs is shown in Figure 3. From the figure, $P_{b}, P_{b_{i}}$ and $P_{b_{o}}$ represents the overall, inner UEs and outer UEs probabilities respectively. The outer UEs suffer from much higher call blocking probability than the inner UEs. This is due to the fact that the outer channel utilization is higher for the outer UEs than the inner channel utilization for the inner UEs.

Figure 4 and Figure 5 show the outage probabilities of the inner UEs and outer UEs respectively for different values of $\mathrm{PR}$ with respect to different values of the offered load. With no PC scheme, it can be seen that the outer UEs suffers from high outage in Figure 5 compared to that of inner UEs shown in Figure 4. Although there is an increase in inner UEs outage of about $3.8 \%$ but since there is significant decrease in the outage for outer UEs with PC scheme, we still believe that our scheme performs better. From Figure 5, it can clearly be seen that with no PC scheme (i.e. PR = 1 ), the outer UEs outage is about $0.36(36 \%)$ where as with PC scheme, the outage is decreased to about

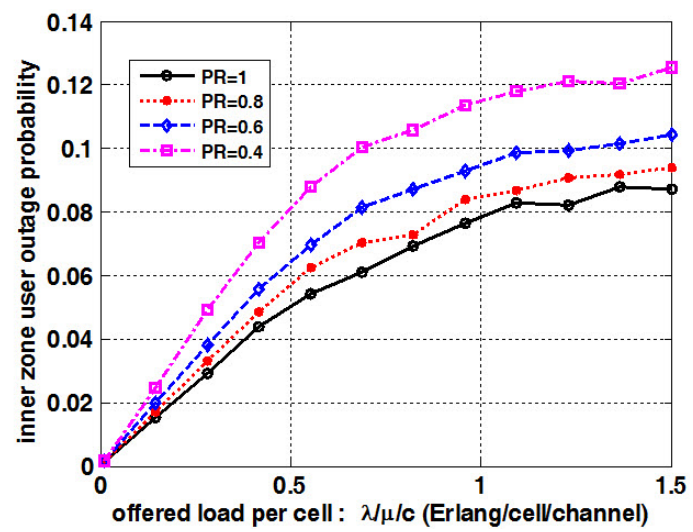

Fig. 4. Inner UEs outage versus offered load at different power ratio (PR).

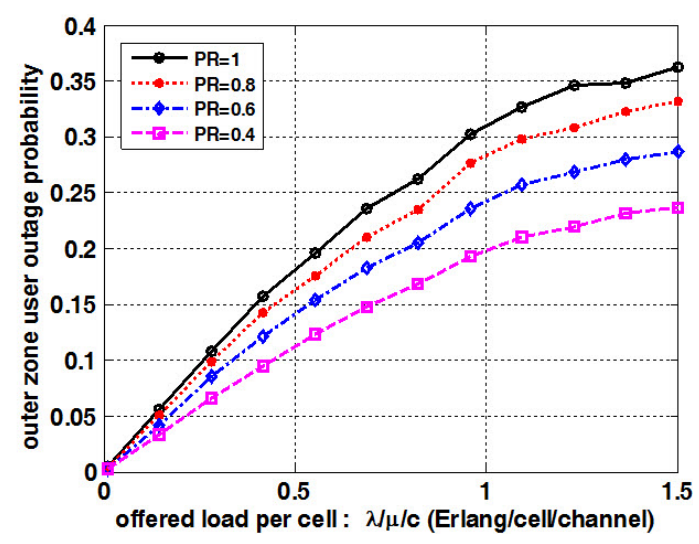

Fig. 5. Outer UEs outage versus offered load at different power ratio $(\mathrm{PR})$

$0.23(23 \%)$.

Fig. 6 shows the outage probabilities for different values of PR with respect to different values of the offered load. From this figure, it was observed that the outage probability with no PC scheme (i.e. $P R=1$ ) was higher compared to the ones with PC scheme. There was further decrease of the outage in the system with PR decrease as it can be seen that the lowest outage was found at $P R=0.4$ for all values of $r$.

Figure 7 shows the relationship between the effective throughput and the outage probability with respect to different values of PR (i.e., $P R=1.0,0.8,0.6$ and 0.4). Figure 7 shows that the effective throughput can not be increased without not only increasing the tolerance of the outage probability for all users but also decreasing the $P R$. For example, the system can 


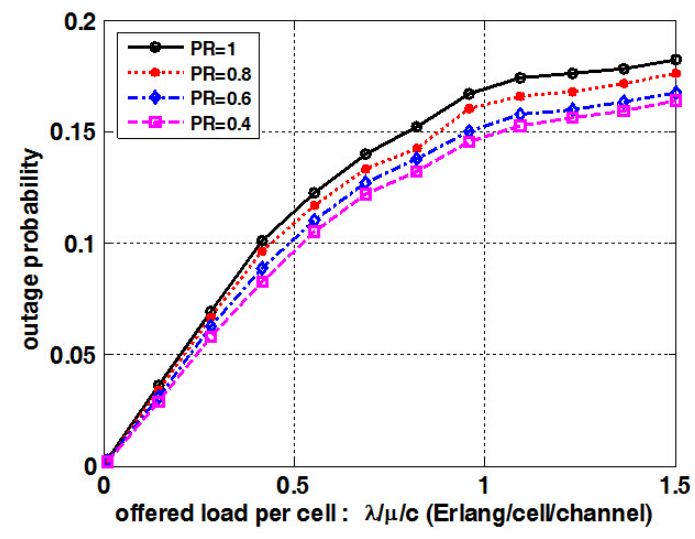

Fig. 6. Outage probability versus offered load at different power ratio $(\mathrm{PR})$.

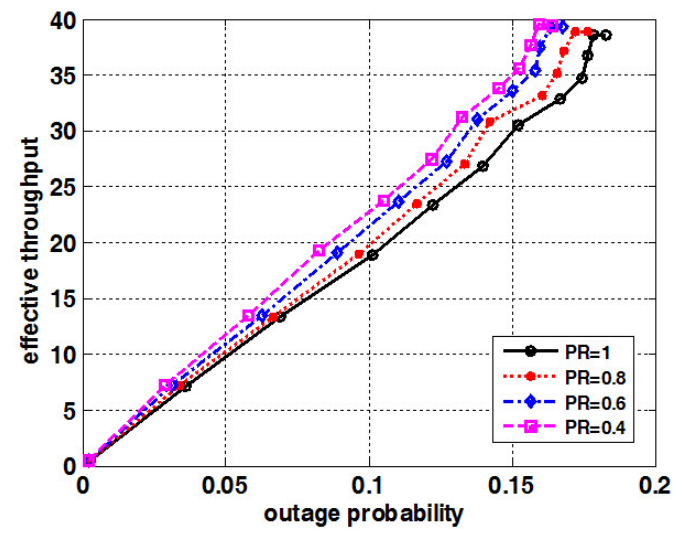

Fig. 7. Effective throughput versus Outage probability at different power ratio (PR).

reach the effective throughput of over 8 with the outage probability of 0.15 for $P R=0.4$ and with the outage probability of 0.17 for $P R=1$.

For the extreme values of normalized inner radius $r . r=0$ means that $\lambda_{i}=0$ and $\lambda_{o}=\lambda$, hence the system efficiency is solely from the external UEs. $r=1$ means that $\lambda_{i}=1$ and $\lambda_{o}=0$, hence the whole cell area is solely covered by inner UEs and hence the system performance is only from inner UEs. In either case of $r$ at this point, the system performance is constrained to only one type of user and that the UEs will transmit with the same power i.e. $P_{t}^{i}$ or $P_{t}^{o}$, for UEs in the inner or outer regions respectively. Accordingly, the $\mathrm{PC}$ scheme results agree with the results of no PC scheme for each extreme value of $r$.

\section{Conclusion and Future Work}

In this paper, we investigated the performance of the cellular network using power control based FRP scheme. From the simulation results, it was observed that the system performance depends not only on the offered load but also on both the size of the inner zone and BS transmit power. With PC scheme showed significant decrease in the outage experienced by the outer UEs which led to the system performance improvement as compared to its counterpart i.e. no PC scheme. This significant improvement was found to occur at power ratio (PR), $\varepsilon=0.4$ for all values of $r$. For better performance for the system of this kind, as it was seen from simulation results, the size of the inner zone and the power ratio are recommended to be 0.7 and 0.4 respectively for high offered load. For low offered load systems, inner zone size of 0.8 is recommended for the same power ratio.

This paper presents only the inner zone ratio and the power ratio that give the optimum system capacity. The future work of this paper is to utilize our results in [8-9] and compare the performance.

\section{References}

[1] S. Kim, H. Lee, and S. Ryu, "Analytical evaluation of almost blank subframes for heterogeneous networks," J. KICS, vol. 38 no. 04, Apr. 2013.

[2] S.-J. Lee, S.-Y. Kim, H.-W. Lee, S.-W. Ryu, and C.-H. Cho, "Adaptive power control schemes for interference mitigation in LTE femtocell networks,” J. KICS, vol. 37, no. 6, Aug. 2012.

[3] G. Boudreau, J. Panicker, G. Ning, C. Rui, and W. Neng, "Interference coordination and cancellation for 4G networks," IEEE Commun. Mag., vol. 47, no. 4, pp. 74-81, Apr. 2009.

[4] Inter-cell interference handling for E-UTRA. R1-050764, 3GPP TSGRAN WG1 Meeting 42, 2005.

[5] N. Himayat, S. Talwar, A. Rao, and R. Soni, "Interference management for $4 \mathrm{G}$ cellular 
standards [WiMAX/LTE update]," IEEE Commun. Mag., vol. 48, no. 8, pp. 86-92, Aug. 2010.

[6] S. W. Halpern, "Reuse partitioning in cellular systems," in Proc. IEEEVTC, pp. 322-327, May 1983.

[7] S.-Y. Kim, S. Ryu, C.-H. Cho, and H.-W. Lee, "Performance analysis of a cellular network using frequency reuse partitioning," Performance Evaluation, vol. 70, no. 2, pp.7789, Feb. 2013.

[8] L. Dong, Z. Song, L. Wenxin, and W. Wenbo, “A frequency reuse partitioning scheme with successive interference cancellation for OFDMA uplink transmission," in Proc. IEEE PIMRC, pp. 1362-1366, Sept. 2009.

[9] S. Meng, S. Kai, H. Wei, X. Yi, and W. Meiting, "An improved fraction frequency reuse with power control in OFDMA cellular systems," in Proc. IEEE TENCON, pp. 1-5, Oct. 2013.

[10] IEEE standard for local and metropolitan area networks, Part 16: Air interface for fixed and mobile broadband wireless access systems, Feb. 2006.

[11] S.-Y. Kim, H.-Y. Lee, and S.-W. Ryu, "Analysis of call admission control for joint transmissionbased LTE- advanced systems," J. KICS, vol. 38, no. 07, Jul. 2013.
무스타파 (Mustafa Habibu Mohsini)

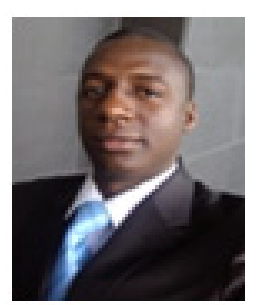

2009년 12월 : University of

Dares Salaam, Tanzania

(BSc. in Computer Engineering \& IT)

2010년 3월 2014년 2월 : Tutorial

Assistant, DoCEA Dept., University of Dodoma (UDOM), Tanzania.

2014년 2월 : 고려대학교 컴퓨터 정보학과 석사

2014년 3월 현재 : Assistant Lecturer, DoCEA Dept., UDOM, Tanzania (On Study Leave)

2014년 9월 현재 : 고려대학교 컴퓨터 정보학과 (박 사과정)

<관심분야>Network Traffic Management, Wireless

Communication Systems, 3GPP LTE-A HetNets

\section{김 승 연 (Seung-Yeon Kim)}

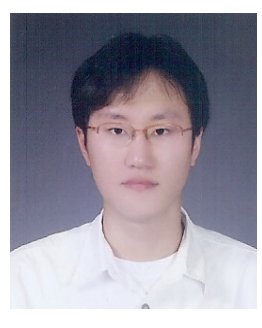

2005년 2월 : 고려대학교 전자

및 정보공학부 졸업

2007년 2월: 고려대학교 전자 정보공학과 석사

2012년 8월: 고려대학교 전자 정보공학과 박사

2015년 현재 : 고려대학교 컴퓨

터 정보학과 교수

<관심분야> 통신망 설계 및 성능 분석, $\mathrm{MAC}$ 프로 토콜

조 충 호 (Choong-Ho Cho)

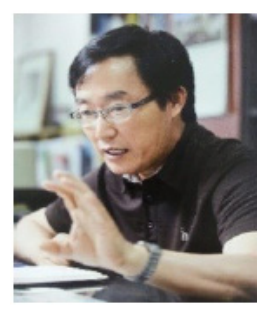

1981년 2월 : 고려대학교 산업 공학과 졸업

1993년 2월: 고려대학교 산업 공학과 석사

1986년 : 프랑스 INSA de Lyon 전산학과(석사)

1989년 : 프랑스 INSA de Lyon 전산학과(박사)

1990 1994년 : 순천향대학교 전산통계학과 조교수

1994 현재 : 고려대학교 컴퓨터정보학과 교수

<관심분야> 통신망 트래픽 관리기술, 무선통신 시

스템, 멀티미디어통신, 인터넷 비즈니스 